\title{
Fracture of a Colonic Self-expandable Metallic Stent in Malignant Colonic Obstruction
}

\author{
Akinari Takao ${ }^{1}$, Taku Tabata ${ }^{2}$, Koichi Koizumi ${ }^{1}$, Go Kuwata ${ }^{1}$, Satomi Shibata ${ }^{1}$, \\ Makiko Mori ${ }^{1}$, Kazuro Chiba ${ }^{1}$, Sawako Kuruma ${ }^{1}$, Tomoko Onishi ${ }^{1}$, Takashi Fujiwara ${ }^{1}$, \\ Terumi Kamisawa ${ }^{1}$, Junko Fujiwara ${ }^{2}$, Takeo Arakawa ${ }^{2}$, Kumiko Momma ${ }^{2}$, \\ Tatsu Shimoyama ${ }^{3}$ and Keiichi Takahashi ${ }^{4}$
}

\begin{abstract}
:
Self-expandable metallic stents (SEMSs) are used for the management of malignant colorectal obstruction. A patient who underwent colonic uncovered SEMS insertion for extraluminal stenosis in the splenic flexure of the transverse colon due to advanced gastric cancer is herein reported. The patient presented with a fracture of the colonic SEMS 494 days after SEMS insertion. Although various complications of stenting have previously been reported, the details of fractures of colonic SEMSs have not yet been reported. Because the improvement in the prognosis for patients who undergo palliative SEMS insertion leads to long-term SEMS placement, diverse complications can thus be expected, and new events like stent fracture are expected to increase in the future.
\end{abstract}

Key words: self-expandable metallic stent, colon, complication, palliative, fracture

(Intern Med 57: 329-332, 2018)

(DOI: 10.2169/internalmedicine.9023-17)

\section{Introduction}

Many recent studies have explored the clinical outcomes and complications in patients who have undergone selfexpandable metallic stent (SEMS) insertion for malignant colorectal obstruction. Since 2012, SEMSs have been used for the management of malignant colorectal obstruction in Japan as either a bridge to surgery (BTS) or as palliative therapy. SEMS insertion is a very useful and reliable procedure for patients with malignant colorectal obstruction since this procedure helps such patients to maintain their quality of life. On the other hand, serious complications, such as perforation, migration, and re-obstruction, sometimes occur.

A case of a fractured colonic SEMS during long-term placement for colonic obstruction due to advanced gastric cancer is herein presented. Although various complications of stenting have previously been reported, the details of fractures of colonic SEMSs have not yet been reported. This accidental complication suggests a clinical approach to the long-term management of colorectal SEMSs. An increasing number of patients are currently undergoing long-term SEMS placement, and such complications are expected to become more diverse in the future.

\section{Case Report}

A 65-year-old woman was admitted to our hospital in July 2013 due to nausea and lower abdominal pain lasting for 1 month. Her surgical history included operations for an ovarian cyst and for lung cancer. Upper gastrointestinal endoscopy and a barium enema examination showed advanced primary gastric cancer. Abdominal computed tomography (CT) showed the presence of extraluminal stenosis at the splenic flexure of the transverse colon due to peritoneal dissemination from gastric cancer. Because the gastric cancer

\footnotetext{
${ }^{1}$ Department of Gastroenterology, Tokyo Metropolitan Komagome Hospital, Japan, ${ }^{2}$ Department of Endoscopy, Tokyo Metropolitan Komagome Hospital, Japan, ${ }^{3}$ Department of Oncology, Tokyo Metropolitan Komagome Hospital, Japan and ${ }^{4}$ Department of Surgery, Tokyo Metropolitan Komagome Hospital, Japan

Received: February 14, 2017; Accepted: May 11, 2017; Advance Publication by J-STAGE: October 16, 2017

Correspondence to Dr. Akinari Takao, aki-tko@cick.jp
} 


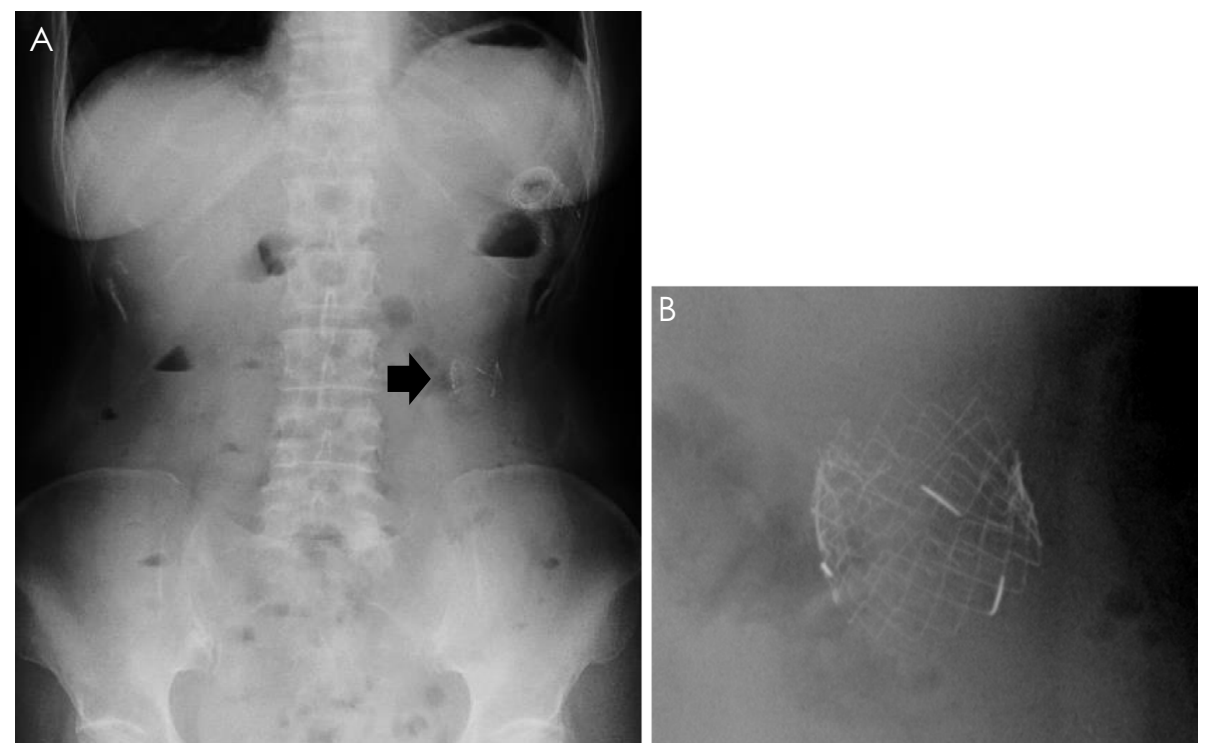

Figure 1. Abdominal X-ray 16 months after SEMS insertion showing a shortened colonic stent at the splenic flexure of the transverse colon (A). A metallic piece of ruptured stent is detected in the proximal colon (B). SEMS: self-expandable metallic stent

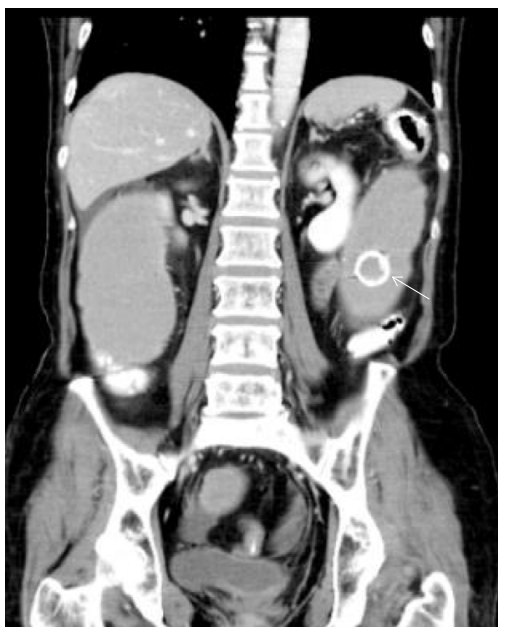

Figure 2. CT shows bowel obstruction at the splenic flexure of the transverse colon and a stent fragment in the distended proximal transverse colon.

was diagnosed to be unresectable, a colonic SEMS (Niti-S Enteral Colonic Uncovered Stent ${ }^{\circledR}$, D-type $22 \mathrm{~mm} / 8 \mathrm{~cm}$; Taewoong, Gimpo, South Korea) was inserted to relieve the bowel obstruction at the splenic flexure of the transverse colon. The patient's clinical symptoms improved soon after stenting; chemotherapy for gastric cancer with S-1 plus cisplatin was started in August 2013.

In September 2014, the patient was re-admitted to our hospital due to nausea and abdominal pain. An abdominal $\mathrm{X}$-ray examination showed a shortened stent at the splenic flexure of the transverse colon and a piece of the metallic stent on the proximal side of the obstruction (Fig. 1). Abdominal CT showed the presence of bowel obstruction at the splenic flexure of the transverse colon caused by the reobstruction of the SEMS and the fracture of the SEMS con-

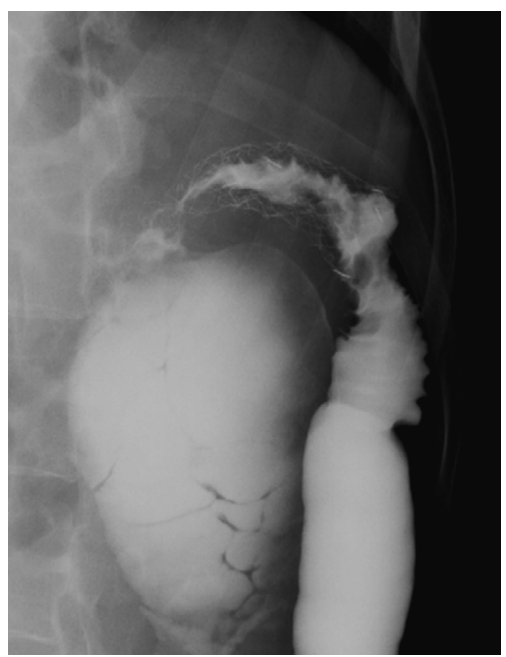

Figure 3. Barium enema shows the narrowing of the SEMS due to tumor ingrowth and the occlusion of the SEMS due to tumor overgrowth. SEMS: self-expandable metallic stent

taining a stent fragment in the proximal dilated transverse colon (Fig. 2). A barium enema examination showed a narrowing of the SEMS due to tumor ingrowth and occlusion of the SEMS due to tumor overgrowth (Fig. 3).

In order to treat this re-obstruction and fracture of the SEMS, a two-stage endoscopic approach was performed. First, another SEMS (Niti-S Enteral Colonic Uncovered Stent $^{\circledR}$, D-type $22 \mathrm{~mm} / 12 \mathrm{~cm}$ ) was inserted through the previous SEMS as a stent-in-stent. Second, endoscopic retrieval of the fragment of the fractured stent was attempted 6 days after the stent-in-stent procedure. The fragment was successfully retrieved through the over-tube by grasping it with a snare, thereby avoiding damage to the SEMS and normal mucosa (Fig. 4). After completion of this treatment, the pa- 

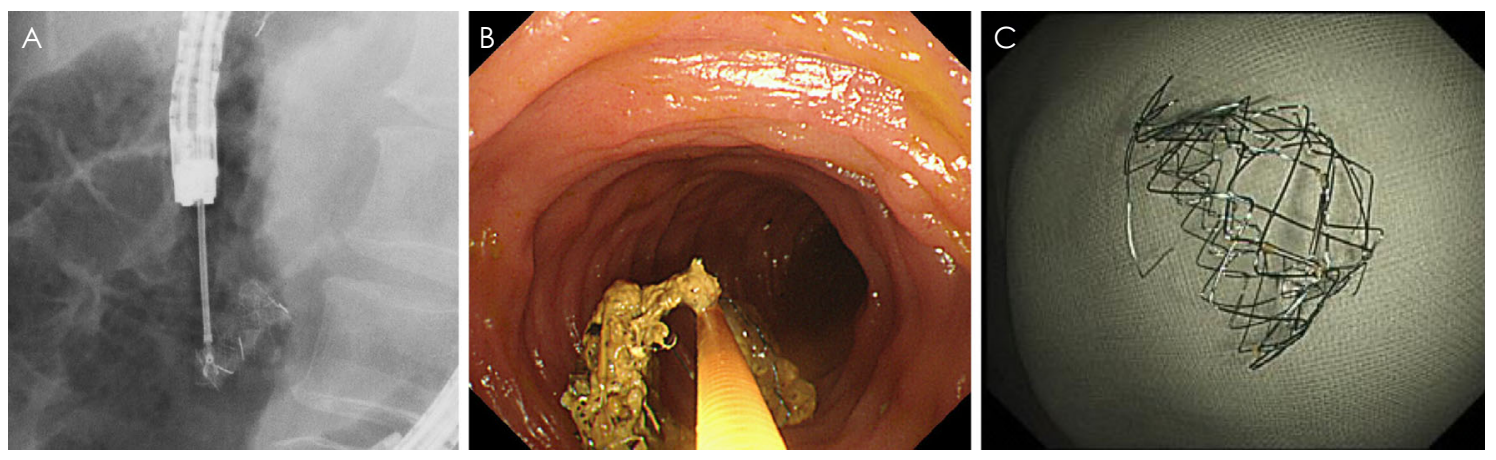

Figure 4. An intertwined fragment is endoscopically retrieved by using a biopsy forceps (A, B). The metallic piece of the ruptured stent is successfully removed (C).

tient's clinical symptoms were relieved, and she was discharged. Chemotherapy for gastric cancer was then restarted in June 2015. Currently (June 2016), two years have passed since the first stent placement, and the patient is still alive.

\section{Discussion}

Although colorectal stenting is a useful procedure in patients with malignant colorectal obstruction, various complications have been reported in previous studies. A prospective multicenter study showed major complications related to colorectal stent placement such as perforation (2.1$5.2 \%)(1,2)$, stent migration $(1.0-11.8 \%)(1,3)$, and reobstruction (18\%) (4). Other minor complications, such as fever $(1.4 \%)(1)$, abdominal pain $(0.2-1.2 \%)(1,2)$, and bleeding $(0.4-5.0 \%)(2,5)$, have also been reported. Several reports have shown stent fractures of biliary $(6,7)$, esophageal $(8)$, and duodenal stents $(9,10)$. On the other hand, the details of fractures of colorectal stents have not yet been reported in the literature; the present case is the first such report and suggests a clinical approach to the long-term management of colorectal SEMSs.

In the present case, two main causes of stent fracture were suspected in hindsight. First, long-term SEMS placement seems to be related to stent fracture. In most palliative cases with colorectal malignant obstruction, SEMS placement normally lasts less than 6 months due to the typically poor prognosis in such patients. On the other hand, as remarkable advances in chemotherapy improve the patients' prognoses, SEMS placement tends to be for longer than a year in some cases. Yoshida et al. reported that, for palliative SEMS, the median duration of patency was $340 \pm 65.5$ days, and the mean survival time after stent insertion was $240.1 \pm 39.1$ days (11). In the present case, 494 days had passed after the first SEMS insertion until the fragment of SEMS was detected in the proximal transverse colon. The deterioration of the SEMS in vivo during long-term insertion could be a factor contributing to stent fracture. Second, the location of the SEMS insertion could have been related to the stent rupture in the present case. Complications, such as perforation and kinking, are more frequently seen in patients in whom the SEMS is inserted at a flexure, such as the sig- moid colon and hepatic or splenic flexure. SEMSs are subject to stronger shearing forces in the flexure because of peristalsis and respiratory movement, and metal fatigue due to prolonged frictional forces could be a factor in such stent fracturing. Fractures of metallic stents have previously been described with biliary $(6,7)$, esophageal (8) and gastroduodenal stents $(9,10)$. In the biliary and esophageal cases, thermal straining such as radiation therapy or electromechanical effort could have result in stent fracture. Fractured gastroduodenal stents also resulted from repeated and prolonged shearing forces on the stent due to anatomic factors.

In the present case, a Niti-S colonic stent was used at the splenic flexure. The stent was made from hand-woven nitinol wire mesh and had low axial force with each cell acting as a cushion. The prolonged friction between the stenotic section and the non-stenotic section of the stent might have triggered the fracture of the colonic SEMS. Although the Niti-S colonic stent is said to be suitable for stenosis in flexures of the colon, it can be presumed that the inappropriate length and low axial force of the stent at the flexure led to stent fracture in this case.

Recently, combination therapy with colorectal SEMSs and chemotherapy has shown good outcomes and has also improved the median survival time, as well as the quality of life, in palliative cases with malignant colorectal obstruction. Since the prolongation of survival in patients who undergo palliative SEMS insertion leads to long-term SEMS placement, the types of complications are expected to diversify, and new events like stent rupture can thus be expected to increase in the future.

In conclusion, colorectal stents can fracture during longterm placement, and we must keep in mind that unexpected complications can occur during long-term SEMS placement. We should routinely check SEMS patency and the SEMS position once every few months on X-ray examinations, even if there are no symptoms to suggest any complications. Further reports are needed to identify the complications and safety issues associated with the long-term placement of colorectal SEMSs.

The authors state that they have no Conflict of Interest (COI). 


\section{References}

1. Matsuzawa $\mathrm{T}$, Ishida $\mathrm{H}$, Yoshida $\mathrm{S}$, et al. A Japanese prospective multicenter study of self-expandable metal stent placement for malignant colorectal obstruction: short-term safety and efficacy within 7 days of stent procedure in 513 cases. Gastroinst Endosc 82: 697-707, 2015.

2. Meisner S, González-Huix F, Vandervoort JG, et al. Selfexpanding metal stenting for palliation of patients with malignant colonic obstruction: effectiveness and efficacy on 255 patients with 12-month's follow-up. Gastroenterol Res Pract 2012: 296347, 2012.

3. Sebastian S, Johnston S, Geoghegan T, et al. Pooled analysis of the efficacy and safety of self-expanding metal stenting in malignant colorectal obstruction. Am J Gastroenterol 99: 2051-2057, 2004.

4. Lee JM, Byeon JS. Colorectal stents: current status. Clin Endosc 48: 194-200, 2015.

5. Khot UP, Lang AW, Murali K, et al. Systematic review of the efficacy and safety of colorectal stents. Br J Surg 89: 1096-1102, 2002.
6. Alkhiari R, Pate V, Cohen L, et al. Spontaneous fracture of a covered self-expandable biliary metal stent and endoscopic technique for removal. Can J Gastroenterol Hepatol 28: 411-412, 2014.

7. Joo KR, Paik CN, Chung WC, et al. Fracture of self-expandable metal stent during endoscopic removal in benign bililary stricture. Clin Endosc 46: 95-97, 2013.

8. Wadsworth CA, East JE, Hoare JM. Early covered-stent fracture after placement for a benign esophageal stricture. Gastroinst Endoc 72: 1260-1261, 2010.

9. Stern N, Smart H. Repeated enteral stent fracture in patient with benign duodenal stricture. Gastroinst Endoc 72: 655-657, 2010.

10. Javaid MR, Yusuf AM. An instant rare complication: a fractured metallic pyloric stent. BMJ Case Rep 2013.

11. Yoshida $\mathrm{S}$, Watabe $\mathrm{H}$, Isayama $\mathrm{H}$, et al. Feasibility of a new selfexpandable metallic stent for patients with malignant colorectal obstruction. Dig Endosc 25: 160-166, 2013.

The Internal Medicine is an Open Access article distributed under the Creative Commons Attribution-NonCommercial-NoDerivatives 4.0 International License. To view the details of this license, please visit (https://creativecommons.org/licenses/ by-nc-nd/4.0/).

(C) 2018 The Japanese Society of Internal Medicine Intern Med 57: 329-332, 2018 